\title{
COMMENTARY
}

\section{COVID-19 and patient engagement in health research: What have we learned?}

\author{
Simon Denegri BA, Bella Starling PhD
}

- Cite as: CMAJ 2021 July 12;193:E1048-9. doi: 10.1503/cmaj.210998

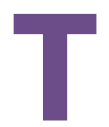

here may never be a better time to take stock of recent developments in patient engagement than during the COVID-19 global pandemic. Science has been front-ofmind in the public consciousness, and scientific conduct has been open to scrutiny in an unprecedented way. Barriers to digital forms of engagement that once seemed impossible have now become mere hurdles to jump. Volunteer participation in clinical trials has propelled COVID-19 research, including development of vaccines at record speed, and serves as a reminder of people's capacity for research altruism. ${ }^{1}$ Yet the pandemic has also highlighted the distance still to be travelled before we can be sure that "nothing about us without us" is more than just rhetoric when it comes to decision-making in health care and research. ${ }^{2}$ Racialized communities and marginalized groups have experienced disproportionately detrimental health and wellbeing effects of the pandemic after long-term neglect by health systems, ${ }^{3}$ and some have noted a catastrophic failure to coproduce COVID-19 strategies tailored to these communities. ${ }^{4}$

In recent years patient engagement has assumed an increasingly prominent place on the agendas of many national health care and research systems. Patient and public involvement in research has evolved into a global movement, with increasingly strong community engagement research in the Global South, ${ }^{5}$ and some countries - such as the United Kingdom, Canada and Ireland - going so far as to establish publicly funded infrastructure to support its development. In 2019, more than 250 organizations and individuals - from Australia to Zimbabwe - joined the first ever global network to strengthen patient engagement around the world. ${ }^{6}$ The persistent and constructive activism of articulate and well-informed patient advocates has led to wide acknowledgement of the importance of patient engagement in tackling current and future health challenges, if not its actualization. However, as Biddle and colleagues noted with regard to Europe, "the implementation of PPI [patient and public involvement] is highly uneven, and PPI is not yet firmly embedded or adequately formalised in European healthcare systems and research, possibly due to a lack of infrastructure, guidance and support."

The fragility of patient engagement in research - even in those regions of the world where it is most developed - became dramatically clear in the early days of the COVID-19 pandemic, when researchers defaulted all too easily to excluding the

\section{KEY POINTS}

- Patient engagement has occupied an increasingly prominent place on the agenda of health care and research systems in recent years.

- The COVID-19 global pandemic has highlighted both the fragility of patient engagement in research and innovative developments in this sphere.

- Better digital engagement of patient groups has removed some of the long-standing barriers to patient and public engagement such as geography, inaccessible environments and cost, enabling new kinds of collaboration.

- The research community including patients must build on the lessons learned during COVID-19 to strengthen the foundation for patient engagement in research and policy.

patient voice in research by dropping patient engagement. A UK Health Research Authority study found that in March 2020 - the month in which the first lockdown began in the UK - the number of research studies in which patients were involved fell from $80 \%$ to $22 \% .{ }^{8}$ When asked, many researchers said that the need to set up studies rapidly prevented or removed the usual need for patient engagement in its design, conduct or review, a view that was shown to be based on incorrect assumptions of patient groups' actual capacities and readiness for involvement. Given the daily public conversation about science and the need for public co-operation to stem the tide of the pandemic, governments' failures to actively involve the public in decisions about pandemic strategies represent missed opportunities for meaningful policy-making. After all, evidence suggests that trust in scientists is a key determinant of whether people will adapt their behaviours to reduce infection, ${ }^{9}$ and what better way to gain public trust than by involving the public?

The pandemic has also presented new opportunities and learnings for patient engagement. Just as regulatory committees have shown their agility in swiftly approving urgent studies, public involvement groups have risen to the challenge of working effectively at speed. For example, the UK Health Research Authority, after noting the aforementioned dramatic drop-off in patient involvement, established an innovative and rapid national "matching" service to connect researchers with patients 
and members of the public willing to engage in partnerships. Most researchers who used the service were new to working in partnership with patients and the public. Many patient engagement groups also showed themselves to be adept at overcoming the challenges of lockdown to continue collaborating with their research partners. And the patient activism that led to the naming and study of Long COVID should not forgotten. ${ }^{10}$

Perhaps most important of all, increased use of digital platforms has removed some of the long-standing barriers to patient and public engagement, such as geography, inaccessible environments and cost, and has enabled global conversations in a way that was not possible before. Enforced it may have been, but virtual working and digital connection have powerfully enhanced patient engagement, although there is a continued need to work in complementary ways with those who are digitally excluded.

An example of digital connection is Planet DIVOC91, which brought together young people, researchers, artists and designers and patient-engagement methodologists from the UK, India and South Africa to develop a graphic novel. ${ }^{11}$ This creative medium introduced young voices into COVID-19 research priorities and design, and raised awareness that patient engagement is "a thing." Experiences and knowledge were shared and mutual understandings of the pandemic improved, but the project highlights another COVID-19-related learning for patient engagement: there is a need to "fund spaces, not just projects, for ordinary people to come together, dream, create and build power." ${ }^{12}$ As Sanda (a character in the graphic novel) said: "We are all part of the knowing...Champo and I are different, that's why we make a good team. I don't need anyone's permission to be unapologetically me."11

The COVID-19 pandemic has increased the visibility of the value of patient engagement and highlighted the importance of ensuring greater diversity of voices in this sphere. Moreover, it has sparked the development of methods and approaches to address deep-seated barriers, recognize alternate and complementary leadership, and ensure public engagement in governance. As such, the COVID-19 experience should be a wake-up call to all research partners - funders, government, institutions, researchers, patients and others - for closer collaboration in the longer term to ensure that resources are available to place patient engagement on a sounder footing for the future. COVID-19 threatened to be a time of crisis for patient engagement and the valuable lessons learned should not be wasted.

\section{References}

1. Case study: the UK's flagship COVID-19 treatment trial - the RECOVERY trial. London (UK): National Institute for Health Research; 2020. Available: https://www.nihr.ac.uk/case-studies/the-uks-flagship-covid-19-treatment-trial -the-recovery-trial/25358 (accessed 2021 June 6).

2. Murphy X, Tierney E, Ní Shé E, et al.COVID-19: public and patient involvement, now more than ever. HRB Open Res 2020;3:35.

3. Begum N, Verma A, Starling B. How inequalities are affecting the response to COVID-19 [blog]. Manchester (UK): University of Manchester; 2020 Apr. 28. Available: http://blog.policy.manchester.ac.uk/posts/2020/04/how-inequalities -are-affecting-the-response-to-covid-19/ (accessed 2021 June 6).
4. Williams $\mathrm{O}$, Tembo D, Ocloo J, et al. Co-production: working together for a fairer future [blog]. Bristol (UK): Bristol University Press/Policy Press; 2021 May 24. Available: https://www.transformingsociety.co.uk/2021/05/24/co-production -working-together-towards-a-fairer-future/ (accessed 2021 June 6).

5. Tembo D, Hickey G, Montenegro C, et al, Effective engagement and involvement with community stakeholders in the co-production of global health research. BMJ 2021;372:n178.

6. An international network on public involvement in health and social care research. The Cochrane Consumer Network; 2021. Available: https://consumers. cochrane.org/news/international_network (accessed 2021 June 6).

7. Biddle MSY, Gibson A, Evans D. Attitudes and approaches to patient and public involvement across Europe: a systematic review. Health Soc Care Commun 2020 July 23. Available: https://doi.org/10.1111/hsc.13111 (accessed 2021 June 6).

8. Public involvement in a pandemic: lessons from the UK COVID-19 public involvement matching service. London (UK) National Health Service, Health Research Authority; 2021. Available: https://www.hra.nhs.uk/planning-and -improving-research/best-practice/public-involvement/public-involvement -pandemic-lessons-uk-covid-19-public-involvement-matching-service/ (accessed 2021 June 6).

9. Pagliaro S, Sacchi S, Pacilli MG, et al. Trust predicts COVID-19 prescribed and discretionary behavioral intentions in 23 countries. PLoS One 2021;16:e0248334. doi 10.1371/journal.pone.0248334.

10. Callarda F, Peregob E. How and why patients made Long Covid. Soc Sci Med 2021;268:113426.

11. COVID-19 inspired webcomic praised by top scientists and policy makers. Manchester (UK): University of Manchester; 2021 May 7. Available: https:// www.manchester.ac.uk/discover/news/ covid-19-inspired-webcomic-praised -by-top-scientists-and-policy-makers/ (accessed 2021 June 6).

12. Planet DIVOC-91 [website]. Available: https://www.planetdivoc91.com/ (accessed 2021 June 7).

Competing interests: Simon Denegri is an employee of the Academy of Medical Sciences and Bella Starling is an employee of Vocal; both of these institutions funded the DIVOC91 comic that involved young people in understanding and communicating COVID-19 research to their age group. Bella Starling heads a team funded by the National Institute for Health Research and Wellcome Trust. She has received consultant fees for development of the International COVID-19 Data Alliance patient and public involvement and engagement strategy. Bella Starling is also the Chair of the Nuffield Council on Bioethics Enquiry into the Future of Ageing and a trustee of the Society for Neuroacanthocytosis. No other competing interests were declared.

This article was solicited and has not been peer reviewed.

Affiliations: Academy of Medical Sciences (Denegri), London, UK; Vocal Research and Innovation (Starling), Manchester University NHS Foundation Trust; National Institute for Health Research (NIHR) Manchester Biomedical Research Centre (Starling) and NIHR Manchester Clinical Research Facility (Starling), Manchester, Greater Manchester, UK

Contributors: Both authors contributed substantially to the conception and design of the work, drafted and revised it critically for important intellectual content, gave final approval of the version to be published and agreed to be accountable for all aspects of the work.

Content licence: This is an Open Access article distributed in accordance with the terms of the Creative Commons Attribution (CC BY-NC-ND 4.0) licence, which permits use, distribution and reproduction in any medium, provided that the original publication is properly cited, the use is noncommercial (i.e., research or educational use), and no modifications or adaptations are made. See: https://creativecommons.org/licenses/ by-nc-nd/4.0/

Correspondence to: Simon Denegri, Simon.Denegri@acmedsci.ac.uk 